\title{
Effect of amino acid doping on the dielectric properties of triglycine sulphate (TGS) crystals
}

\author{
A. J. Jeyaprakash Manoharan ${ }^{1}$, N. Joseph John ${ }^{1 *}$, V. Revathi ${ }^{2}$, K. V. Rajendran ${ }^{2}$ and P. M. Andavan ${ }^{3}$ \\ ${ }^{1}$ Department of Physics, Government Arts College, Udhagamandalam, Tamilnadu- 643002, India \\ ${ }^{2}$ Department of Physics, Presidency College (Autonomous), Chennai, Tamilnadu- 600005, India \\ ${ }^{3}$ Department of Physics, Government Arts College (Autonomous), Coimbatore, Tamilnadu- 643018, India \\ njosephjohn@gmail.com*
}

\begin{abstract}
Effect of amino acid (proline) doping on the dielectric properties of triglycine sulphate has been studied. Pure and proline doped triglycine sulphate (TGS) were grown from aqueous solution by solution growth technique at room temperature. The dielectric studies were carried out to identify the phase transition temperature and the dielectric behavior. The present study shows that there is an increase in the Curie temperature for the doped crystals.
\end{abstract}

Keywords: TGS crystals, proline doping, dielectric properties.

Introduction

Ferroelectic triglycine sulphate (TGS) is one of the most extensively studied ferroelectric materials, which finds wide application as room temperature IR detector. It also finds application in burglar alarms, medical vidicon tubes operating at room temperature, FTIR instrumentation and in pyroelectric detector. It undergoes a second-order phase transition at Curie temperature $(322 \mathrm{~K})$ from ferroelectric to paraelectric. The crystal structure of TGS is monoclinic below and above Curie temperature (Hoshino et al., 1959). The space group transforms from non-centrosymmetric $P_{21}$ in the ferroelectric phase to centrosymmetrical $P_{21 / m}$ in the paraelectric phase. The cleavage plane is generally expressed as the b-plane and the spontaneous polarization $\left(P_{\mathrm{s}}=2.8 \mu \mathrm{C} / \mathrm{cm}^{2}\right)$ arises along the crystallographic b-axis (Landolt-Bornstein, 1969). However, the TGS has a disadvantage that it depolarizes by thermal, electrical or mechanical means. An efficient way to stabilize the single domain state is practical by doping an optically active molecule into TGS. The practical substitution of an optically active molecule in the place of glycine $d$ molecule causes an internal bias field, which makes the crystal permanently polarized (Nakatani, 1973). Many authors investigated the effect of doping of various amino acids on TGS (Bye et al., 1972; Aravazhi, 1997a,b; Meera et al., 2000). The effect of organic dopants on TGS and rare earth ions dopant with TGS have also been investigated (Mihaylova \& Stoyanov,1996; Meera et al., 2001; Muralidharan et al., 2002; Theresita Shanthi et al., 2009; Selvarajan et al., 2008). In recent years, the interest in studying the pure and doped TGS crystals has increased because of their promise

Research article

CIndian Society for Education and Environment (iSee)
Fig. 1. Photograph showing the pure and proline added TGS crystals grown (from left: pure TGS, 5 \& 10 wt\% proline added)

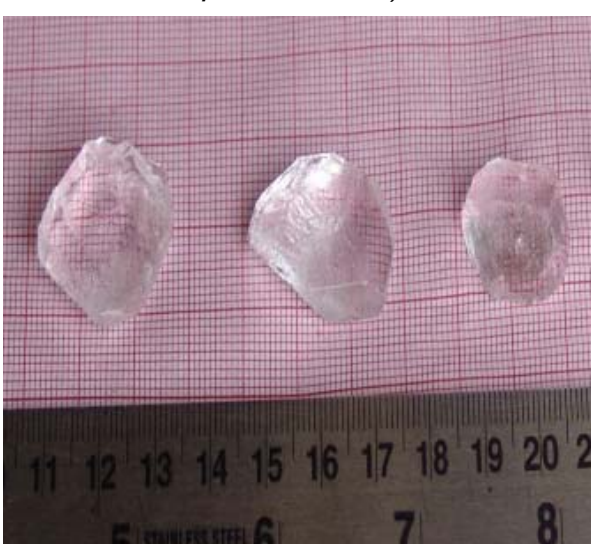

"Triglycine sulphate crystal doping with amino acid" http://www.indjst.org in various devices. In the present work we have chosen the amino acid proline as dopant because proline ions are expected to play a partial role for the spontaneous polarization in TGS crystal thereby increase the dielectric constant and Tc due to its intrinsic dipole moment. The objective of the present work was to investigate the effect of proline addition on the growth and dielectric properties of TGS crystal.

Experimental procedure

Growth of TGS

Triglycine sulphate (TGS) was synthesized (Theresita Shanthi et al., 2009) by taking glycine and sulphuric acid in the molar ratio $3: 1$. Glycine reacts with sulphuric acid as follows:

$3\left(\mathrm{NH}_{2} \mathrm{CH}_{2} \mathrm{COOH}\right)+\mathrm{H}_{2} \mathrm{SO}_{4} \rightarrow\left(\mathrm{NH}_{2} \mathrm{CH}_{2} \mathrm{COOH}\right)_{3} \mathrm{H}_{2} \mathrm{SO}_{4}$

The required amount of sulphuric acid was diluted with doubly distilled water. Then, the calculated amount of glycine was added and dissolved in diluted $\mathrm{H}_{2} \mathrm{SO}_{4}$. The solution was heated until the salt got recrystallized. Extreme care was taken during recrystallization to avoid the oxidation of glycine. Hence, the solution temperature was always maintained below $60^{\circ} \mathrm{C}$. The recrystallized TGS salt was dissolved in doubly distilled water. In this way the natural impurity content of TGS salt was minimized. A saturated solution of TGS at room temperature was prepared using twice crystallized salt. The solution was filtered using a high quality filter paper. The amino acid proline (5 and $10 \mathrm{wt} \%$ ) was added to the saturated solution, till the equilibrium was attained. The solution was kept in glass vessels covered with perforated filter paper for slow evaporation at room temperature. Slow evaporation technique was employed for crystal growth. Good quality transparent crystals were harvested after 25 days. The grown crystals were shown in Fig. 1.

A.J.J.Manoharan et al. Indian J.Sci.Technol. 
The sample crystals cleaved perpendicular to the polar axis with thickness about $4 \mathrm{~mm}$ having graphite coating on the opposite faces was placed between two brass electrode and thus parallel plate capacitor was formed. The capacitance and dielectric loss ( $\tan \delta$ ) were measured using the conventional two probe technique (Joseph John et al., 2007; Joseph John \& Mahadevan, 2008) at various temperatures ranging from 300 to $357 \mathrm{~K}$ using an LCR meter (Model APLAB with two frequencies, namely $100 \mathrm{~Hz}$ and $1 \mathrm{kHz}$ ). The dielectric constant was calculated using the relation:

$$
\varepsilon_{r}=C_{\text {cry }}-C_{\text {air }}\left(1-\left(A_{\text {crys }} / A_{\text {air }}\right)\right) \times\left(A_{\text {air/ }} A_{\text {crys }}\right), C_{\text {air }}
$$

Where, $\mathrm{C}_{\text {crys }}$ is the capacitance with crystal (including air), $\mathrm{C}_{\text {air }}$ is the capacitance of air, $\mathrm{A}_{\text {crys }}$ is the area of the crystal touching the electrode and $A_{\text {air }}$ is the area of electrode. The AC electrical conductivity $\left(\sigma_{\mathrm{ac}}\right)$ was calculated using the relation $\sigma_{\mathrm{ac}}=\varepsilon_{0} \varepsilon_{\mathrm{r}} \omega \tan \delta$ where $\varepsilon_{0}$ is the permittivity of free space $\left(8.85 \times 10^{-12} \mathrm{Farad} / \mathrm{m}\right)$ and $\omega$ is the angular frequency $(\omega=2 \pi f, f=100 \mathrm{~Hz} \& 1 \mathrm{kHz})$.

The resistances of the crystals were measured using a thousand Meg ohmmeter. The observations were made while cooling the sample. The dimensions of the crystals were measured using a traveling microscope. The DC conductivity $\left(\sigma_{\mathrm{dc}}\right)$ of the crystal was calculated using the relation $\sigma_{\mathrm{dc}}=\mathrm{d} /(\mathrm{RA})$ where $\mathrm{d}$ is the thickness of the sample, $A$ is the area of the sample and $R$ is the measured resistance.

\section{Results and discussion}

The term dielectric applies to the material properties governing the interaction between matter and electromagnetic field. Induced or permanent electric polarization or magnetization of matter as a function of static or alternating electric, magnetic or electromagnetic field constitutes the dielectric properties of the material. The dielectric constant is one of the basic electrical properties of solids. Various polarization mechanisms in solids such as atomic polarization of the lattice, orientational polarization of diploes and space charge polarizations can be understood very easily by studying the dielectric properties as a function of frequency and temperature for crystalline solids (Wagner \& Hantemann, 1950; Rao \& Somakula 1965; Govinda \& Rao, 1975) These investigations help in detecting the structural phase transitions taking place in solids when abrupt changes in dielectric properties are observed. Particularly, the presence of a dielectric between the plates of a condenser enhances the capacitance. The effect makes material with dielectric constant useful in capacitor technology.

The magnitude of the dielectric constant depends on the degree of polarization charge displacement in the crystals. The electronic exchange of the number of ions in the crystals gives local displacement of electrons in the direction of applied field that gives polarization. Ferroelectric domains are areas of such local dipole alignment with an associated net dipole moment and net polarization. In presence of an applied electric field, domains that are aligned with the direction of the field will grow at the expense of the less well aligned domains. Thus the observed enhancement in the dielectric constant at low frequency could be attributed to the multidomain state of the proline doped sample. As the frequency increases, a point will be reached where the space charge cannot sustained and comply with the external field giving rise to diminishing values of dielectric constant. Above this frequency the domain wall motion contribution to dielectric constant was nearly the same for pure and doped crystals (Parimalaselvi et al., 2010).

The temperature dependence of dielectric constant $\left(\varepsilon_{\mathrm{r}}\right)$ obtained for the grown crystals for $100 \mathrm{~Hz}$ frequency is provided in Fig.2a and for $1 \mathrm{KHz}$ frequency in Fig. 2b. The temperature dependence of dielectric loss ( $\tan \delta$ ) obtained for the frequency $100 \mathrm{~Hz}$ is presented in Fig.3a and for $1 \mathrm{KHz}$ in Fig. 3b. The dielectric constant and the dielectric loss values increases with the increase in temperature for both the frequencies up to the transition temperature and then decreases. The doped crystals have higher dielectric constant values compared to that of pure TGS. Increase in dielectric constant of doped TGS crystals at transition temperature attributed to free charge carriers created by the dopant.

The Curie point Tc for all the samples was not found to be the same. A small shift in temperature is found for doped TGS crystals. The dielectric constant is small at lower temperature, which increases with temperature and rises sharply up to the Curie point for both the frequencies. Above Tc the dielectric constants decreases suddenly and obey Curie-Wess law. Variation of $\varepsilon_{\mathrm{r}}$ with temperature is generally attributed to the crystal expansion, the electronic and ionic polarizations and the presence of impurities and crystal defects. The variation of $\varepsilon_{\mathrm{r}}$ at low temperature is mainly due to the expansion and electronic and ionic polarization. At higher temperatures, the increase is mainly attributed to the thermally generated charge carriers and impurity dipoles. The AC conductivity shows the same behavior (Fig.4a and $4 b)$.

Fig. 5. shows the temperature dependence of DC conductivity $\left(\sigma_{\mathrm{dc}}\right)$, It can be seen that, for all TGS systems the DC conductivity increases with the increase in temperature. The defect concentration will increase exponentially with temperature and consequently the electrical conduction also increases. The addition of impurity further increases the electrical conduction in the temperature region considered. The conduction region is considered in the present study seems to be connected to mobility of vacancies.

\section{Conclusions}

Single crystals of pure and proline added Triglycine sulphate (TGS) were grown by slow evaporation method. The grown crystals are transparent and with well defined external appearance. Capacitance and dielectric loss
Research article

CIndian Society for Education and Environment (iSee)
"Triglycine sulphate crystal doping with amino acid" http://www.indjst.org
A.J.J.Manoharan et al. Indian J.Sci.Technol. 


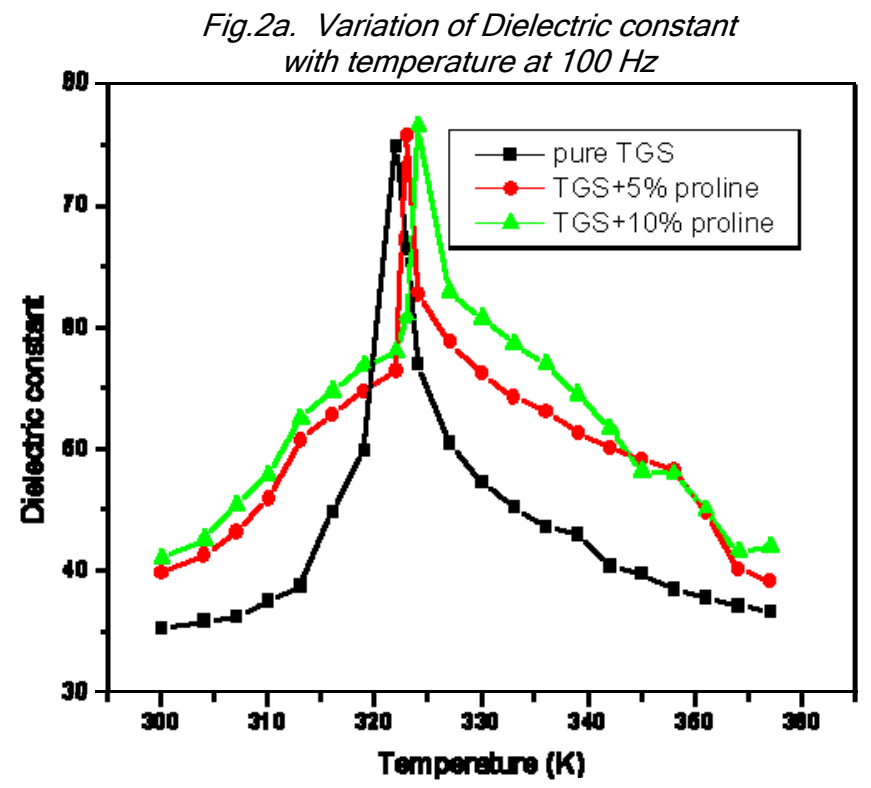

Fig.3a. Variation of Dielectric loss with temperature at $100 \mathrm{~Hz}$

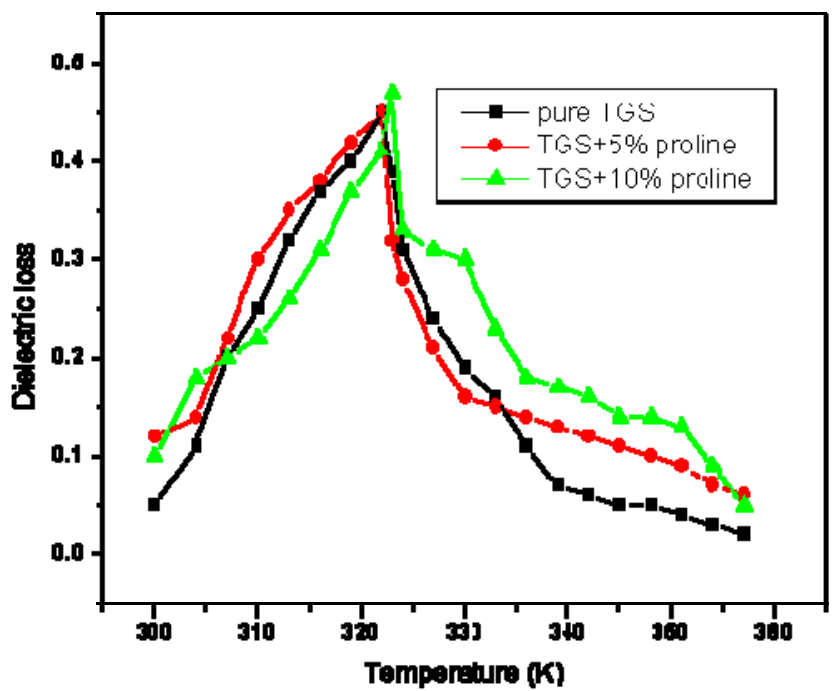

Fig.4a Variation of AC conductivity with temperature at $100 \mathrm{~Hz}$

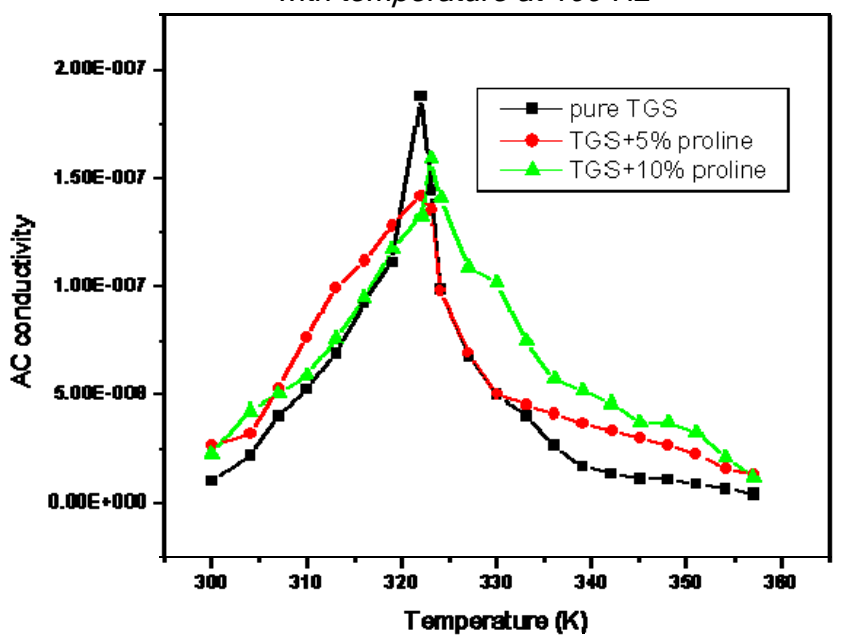

Vol. 4 No. 6 (June 2011)

ISSN: 0974- 6846

Fig.2b. Variation of Dielectric constant with temperature at $1 \mathrm{kHz}$

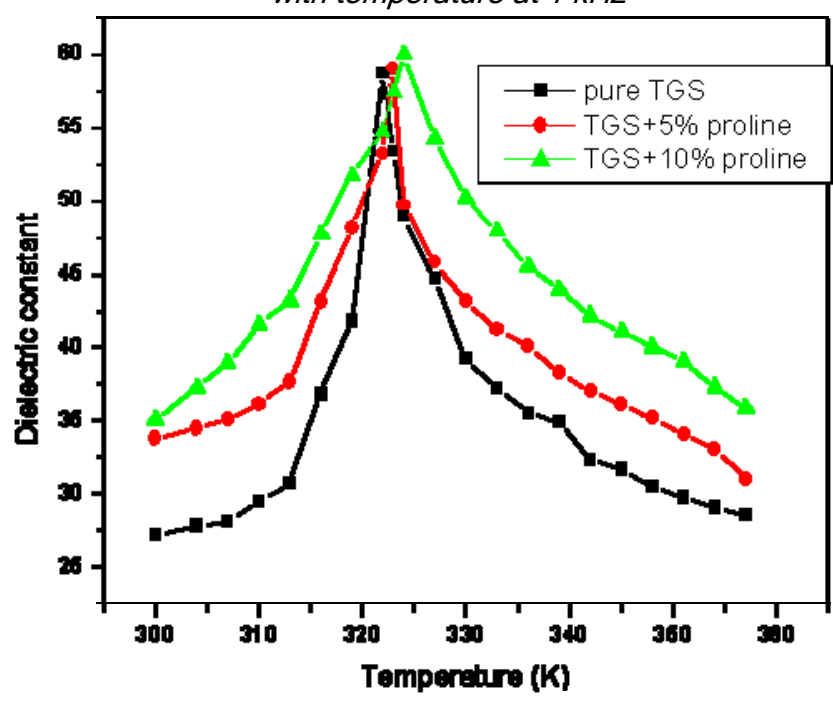

Fig.3b. Variation of Dielectric loss with

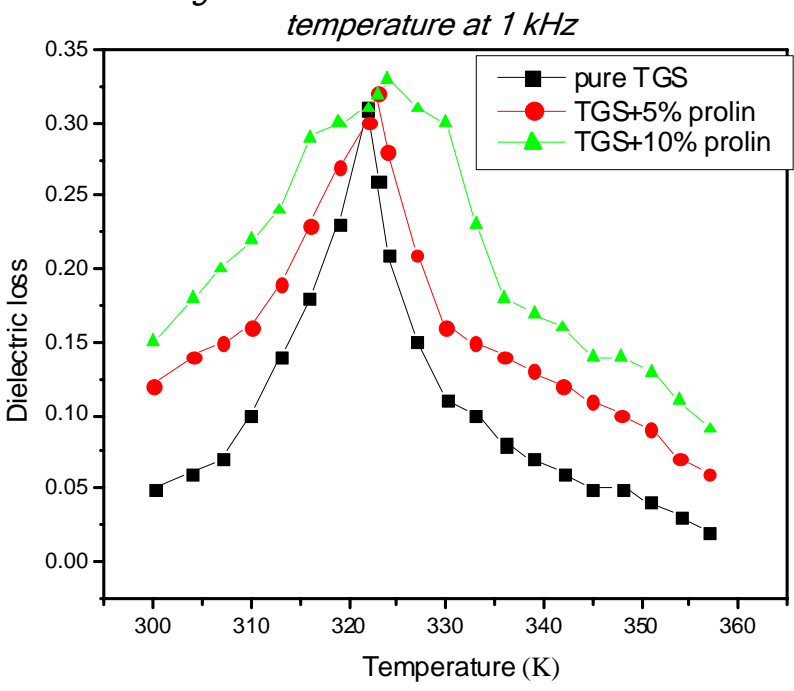

Fig. 4b. Variation of $A C$ conductivity with temperature at $1 \mathrm{kHz}$

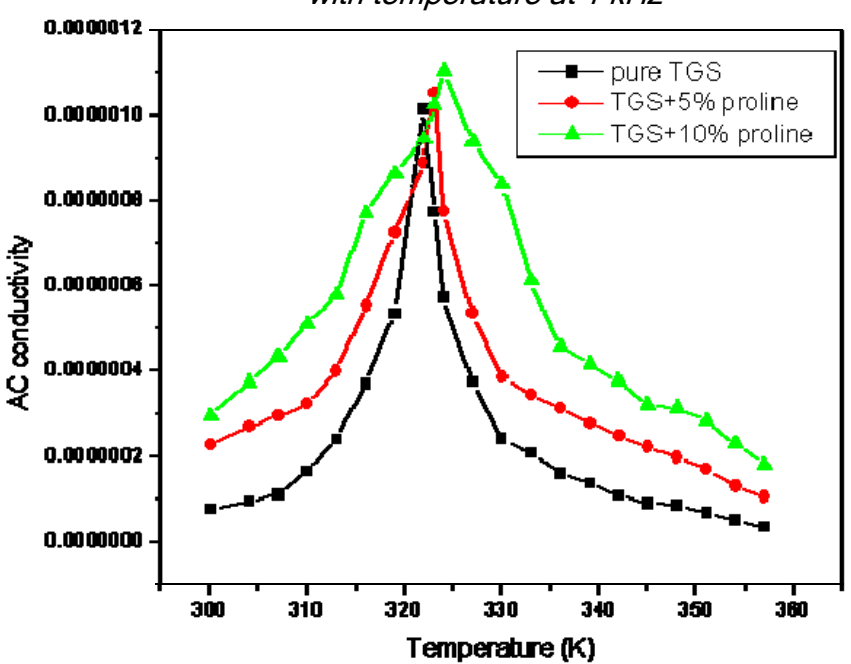

Research article

Clndian Society for Education and Environment (iSee)
"Triglycine sulphate crystal doping with amino acid" http://www.indjst.org
A.J.J.Manoharan et al. Indian J.Sci.Technol. 
Fig.5. Variation of DC electrical conductivity with temperature

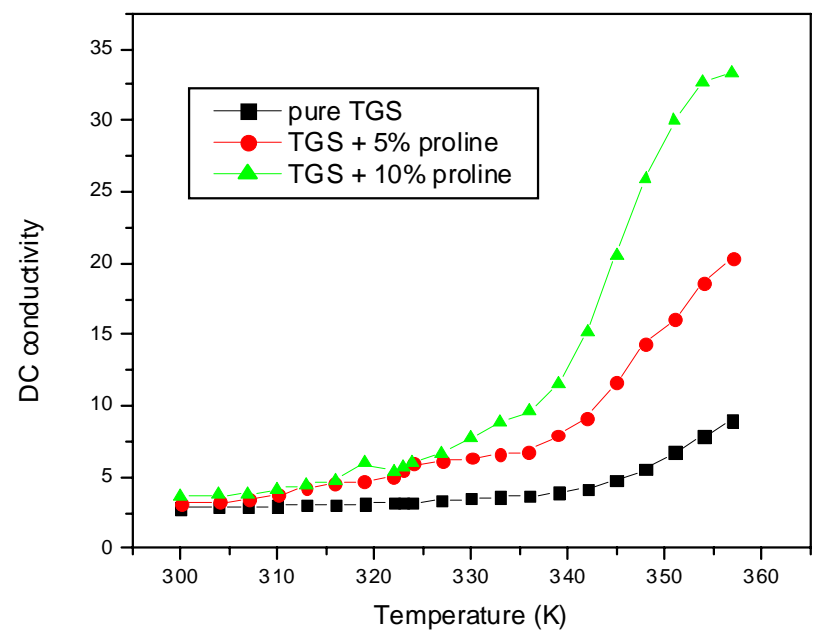

tangent (tan $\delta)$ measurements were carried out for the grown crystals at various temperatures ranging from 300 to $357 \mathrm{~K}$ using an LCR meter with frequencies of $100 \mathrm{~Hz}$ and $1 \mathrm{kHz}$. Dielectric constant and $A C$ electrical conductivity were determined from the measured capacitance and $\tan \delta$ values. The shift in the Curie temperature from 322 to $324 \mathrm{~K}$ in the doped crystals is due to the presence of high polarization capacity of proline. DC electrical conductivity was also measured at various temperatures and found to increase with increasing temperature.

\section{References}

1. Aravazhi S, Jayavel R and Subramanian C (1997a) Growth and stability of pure and amino doped TGS crystals Mater. Chem. Phys. 50, 233-237

2. Aravazhi S, Jayavel R and Subramanian C (1997b) Growth and characterization of L-alanine and L-valine doped triglycine sulphate crystals, Mater. Res. Bull. 32, 1503-1513

3. Bye KL and Keve ET (1972) Structural inhibition of ferroelectric switching in triglycine sulphate. II, XIrradiation/field treatment. Ferroelectrics. 4, 87-95

4. Govinda S and Rao KV (1975) Dielectric properties of single crystals of $\mathrm{Al}_{2} \mathrm{O}_{3}$ and $\mathrm{Al}_{2} \mathrm{O}_{3}$ doped with chromium or vanadium Phys. Stat. Solidi. 27, 639644

5. Hoshino S, Okaya Y and Pepinsky R (1959) Crystal structure of the ferroelectric phase of (Glycine) $)_{3}$. $\mathrm{H}_{2} \mathrm{SO}_{4}$ Phys. Rev. 115, 323-330.

6. Joseph John N and Mahadevan CK (2008) Studies on $\mathrm{NaCl}$ added ADP single crystals Materials \& Manf. Proces. 23, 809-814.

7. Joseph John N, Selvarajan P, Benita Jeba Silviya and Mahadevan CK (2007) Growth and characterization of Disodium Hydrogen Orthophosphate (DSHP) single crystals Materials \& Manf. Proces. 22, 379384.
Vol. 4 No. 6 (June 2011)

ISSN: 0974- 6846

8. Landolt-Bornstein (1969) Crystal and solid state physics. New Series, Group III, vol. 3. Springer, NY. pp: 185.

9. Meera K, Aravazhi S, Santhanaragavan $P$ and Ramasamy P (2000) Growth and characterization of L-tyrosine-doped TGS crystals J. Cryst. Growth. 211, 220-224

10. Meera K, Muralidharan $R$, Santhanaragavan $P$, Gopalakrishnan R and Ramasamy P (2001) Growth and characterization of L-cystine doped TGS crystals J. Cryst. Growth. 226, 303-312

11. Mihaylova E (2000) Raman studies of TGS doped with Nd. J. Phy. Chem. of Solids. 61, 1919-1925.

12. Mihaylova E and Stoyanov St. (1996) Dielectric properties of rare earth doped TGS Phys. Status Solidi. 154, 797-802

13. Muralidharan R, Mohanakumar R, Ushasree PM, Jayavel R and Ramasamy P (2002) Effect of rareearth dopants on the growth and properties of triglycine sulphate single crystals. J. Cryst. Growth. 234, 545-550.

14.Nakatani N (1973) Ferroelectric domain structure of Tri-Glycine sulphate observed using scanning electron microscope. Jpn. J. Appl. Phys. 12, 17231728.

15.Parimaladevi R, Sekar C and Krishnakumar V (2010) The effect of nitric acid $\left(\mathrm{HNO}_{3}\right)$ on growth, spectral, thermal and dielectric properties of triglycine sulphate (TGS) crystal. Spect. Acta Part A. 75, 617-623.

16. Rao KV and Somakula VV (1965) Dielectric properties of cobalt oxide, nickel oxide and their mixed crystals. J. Appl. Phys. 36, 2031-2039.

17. Selvarajan P, Sivadhas A, Freeda TH and Mahadevan CK (2008) Growth, XRD and dielectric properties of triglycine sulphophosphate (TGSP) crystals added with magnesium sulphate. Physica B. 403, 42054208.

18. Theresita Shanthi $T$, Selvarajan $P$ and Mahadevan CK (2009) Growth, structural, mechanical, spectral and dielectric characterization of $\mathrm{NaCl}$-added Triglycine sulphate single crystals. Current Appl. Phys. 9,1155-1159.

19.Wagner C and Hantemann P (1950) Determination of the concentrations of cation and anion vacancies in solid potassium chloride. J.Chem.Phys.18, 72-74.
"Triglycine sulphate crystal doping with amino acid" http://www.indjst.org
A.J.J.Manoharan et al. Indian J.Sci.Technol. 